\title{
Percepción de los estudiantes con respecto a las clases en línea debido al Covid-19 en la Facultad de Contaduría Pública y Administración Campus Cadereyta
}

Flor Elizabeth Carrión García ${ }^{1}$, Janneth Guadalupe Lugo de los Santos² y Samaria Dalila Saldaña Fermín ${ }^{3}$

${ }^{1}$ Universidad Autónoma de Nuevo León, Facultad de Contaduría Pública y Administración Monterrey, Nuevo León, México, lic.florcarrion-facpya@hotmail.com, Av. Universidad S/N Col. Ciudad Universitaria, (+52) 818-168-88-35.

.$^{2}$ Universidad Autónoma de Nuevo León, Facultad de Contaduría Pública y Administración Monterrey, Nuevo León, México, jannlugo@ hotmail.com, Av. Universidad S/N Col. Ciudad Universitaria, (+52) 828294- 24-73

${ }^{3}$ Universidad Autónoma de Nuevo León, Facultad de Contaduría Pública y Administración Monterrey, Nuevo León, México, samaria611@gmail.com, Av. Universidad S/N Col. Ciudad Universitaria, (+52) 828-114-1553

Información del artículo revisado por pares

Fecha de aceptación: junio-2021

Fecha de publicación en línea: diciembre-2021

DOI: https://doi.org/10.29105/vtga7.1-124

\section{RESUMEN}

En la actualidad estamos pasando por una situación en la cual se tiene la preocupación de llevar a cabo las medidas para evitar la propagación del coronavirus COVID -19, por lo cual todos los estudiantes de universidades públicas y privadas están en clases en línea.

El presente trabajo de investigación se lleva a cabo con base a la percepción que tiene los estudiantes respecto a las clases en línea en tiempos de pandemia.

Con la investigación realizada se desea conocer la perspectiva de los alumnos, saber cómo ha afectado de cierto punto en el rendimiento el tener clases en línea, además de conocer el aprovechamiento de las tecnologías de la información usadas en el campus.

PALABRAS CLAVE
Pandemia, clases en línea, docentes, alumnos, COVID -19

\section{ABSTRACT}

We are currently going through a situation in which there is concern to carry out measures to prevent the spread of the COVID -19 coronavirus, for which all students from public and private universities are in online classes.

This research work is carried out based on the perception that students have regarding online classes in times of pandemic.

With the research carried out, it is desired to know the perspective of the students, to know how having online classes has affected a certain point in the performance, in addition to knowing the use of the information technologies used on campus. 


\section{KEYWORDS}

Pandemic, online classes, teachers, students, COVID -19

\section{INTRODUCCIÓN}

En este trabajo se realiza una investigación donde se analiza la importancia que se tiene actualmente en la educación en línea, la pauta que marcará un antes y un después en sistemas educativos mundiales, que es lo que ha ocasionado la crisis sanitaria actual, que hizo que cientos de instituciones educativas cerraran sus puertas como medida precautoria para evitar la propagación del COVID-19. Además de identificar la afectación en el rendimiento educativo y cuál es la actitud de los estudiantes respecto a llevar las clases en línea.

Si bien se sabe que los estudiantes que empezaron a recibir clases en línea, es muy difícil que se mantengan atentos a las clases que brindan los docentes, así como la facilidad con lo que se distraen con muchas cosas y esto al principio de la situación actual del mundo era muy difícil mantener la atención en algún tema o centrarse en lo que se estuviera explicando. Incluso, lo aburrido que se pudiera llegar a ser, hasta un poco tedioso.

A lo mejor para algunos estudiantes; como por ejemplo el nivel básico, aceptan esta nueva forma de llevar sus clases y tal vez algunos de nivel superior que antes ya hayan llevado a cabo este sistema de aprendizaje no se les dificulte o lo disfrutan de igual forma.

Como docentes que somos y que hemos llevado el uso de las herramientas que nos brinda la Universidad Autónoma de Nuevo León, así como el uso de la tecnología y plataformas digitales para poder llevar a cabo nuestras clases a lo largo del período del
2020-2021. Por tal motivo es importante resaltar que hay que hacer las clase entretenidas, divertidas, interesantes y sobre todo que sean dinámicas e interactivas para que el proceso de enseñanza de docente a alumno, así como el aprendizaje alumnodocente sea aún más efectivo.

\section{MARCO TEORICO}

\section{1.- Antecedentes}

Por primera vez en abril del 2020, 30 millones de alumnos regresan a clases de manera virtual, en México, en la Universidad Autónoma de Nuevo León, los 7 mil profesores impartieron sus clases en línea con apoyo de 65 mil aulas virtuales, explotando los recursos tecnológicos con los que contamos; por ejemplo, MS Teams, Nexus.

Trabajar en línea esta de moda en todo el mundo debido al coronavirus.

Es muy difícil trabajar a distancia ya que en materias practicas a veces a los estudiantes no les queda claro el tema, ahora a distancia y frente a una computadora surgen más dudas la mayoría de los docentes y estudiantes queremos regresar a trabajar de manera presencial sin embargo debemos esperar a que las autoridades lo autoricen . La pandemia de enfermedad covid-19, ha provocado que las organizaciones realicen una gestión más eficiente implementando estrategias que se adapten a las tendencias tecnológicas más modernas para el mejoramiento de los procesos internos, debido a que las competencias empresariales demandan que cada vez sea más alta la eficiencia en la toma de decisiones desde el 
liderazgo, es necesario que los líderes provean al equipo de trabajo de las herramientas y direccionamientos necesarios para afrontar los retos que se presentan al momento de administrar una organización. En atención a este propósito se realizó el análisis de Liderazgo Organizacional en los efectos del Covid-19. En la cual se mostrará el planteamiento del problema a investigar, preguntas de Investigación, objetivos de la investigación, justificación, importancia de la investigación, delimitación del estudio, limitaciones del estudio, entre otros puntos.

La educación a distancia surgió a finales del siglo XIX y principios del siglo $\mathrm{XX}$ apoyándose en el desarrollo de la imprenta y de los servicios postales. En sus inicios los programas de formación eran muy simples y estaban basados en textos escritos en los que se reproducían las clases presenciales tradicionales.

La Educación a Distancia es la precursora del e-learning. Y como tal, resulta interesante conocer las diferentes etapas por las que la Educación a Distancia ha ido pasando hasta llegar a la situación actual.

\section{2.- Actualidad}

En el presente año ya se puede conocer a mayor detalle cómo ha afectado la pandemia el rendimiento escolar y la percepción que tienen los estudiantes respecto a llevar a cabo las clases en línea.
"Este es un enorme desafío de equidad educativa que puede tener consecuencias que alteran la vida de los estudiantes vulnerables", dijo Ian Rosenblum, director de The Education TrustNew York. En el cual estoy de acuerdo, sé muy bien que la mayoría de los centros educativos prestan acceso a sitios virtuales, cuentan con material de apoyo en línea y demás recursos, pero la verdad es que, al momento de declararse la pandemia y el retorno de las clases en línea, los sitios de las instituciones no se encontraban preparados para la conectividad de miles/millones de estudiantes. Además de que alumnos tienen la disponibilidad de tener al alcance dispositivos electrónicos, pero no todos cuentan con uno, profesores/docentes tomaron el desafío de adaptarse a la tecnología, diseñar lecciones en línea funcionales para que el aprendizaje sea atractivo para el alumno.

Y bien se sabe que sólo alrededor del $60 \%$ de la población mundial tiene acceso a internet o aun dispositivo; como Tablet, smartphone. Esto ha generado que organismos y las organizaciones que se han unido a esta labor y han aportado su granito de arena para apoyar a los que no cuentan con los recursos necesarios para tener acceso a una red y continuar con sus estudios

"Han sido desalojados de su estabilidad, han sido desalojados de sus hogares, han sido desalojados de su capacidad de vivir de manera cómoda y segura".

En el siguiente articulo nos habla sobre el cierre de escuelas e interrupción de la educación, lo que la pandemia de COVID-19 ha provocado, siendo algo histórico a nivel mundial, esto a su vez teniendo un efecto universal en alumnos y docentes, lo que se ha estado diciendo en artículos anteriores. 
Se sabe que en abril del 2020 la incorporación a clases en línea, el 94 por ciento de los estudiantes de todo el mundo estaban afectados por la pandemia, También se comenta la capacidad de que las instituciones educativas tuvieron para responder al cierre masivo.

\section{Percepción de los estudiantes}

Teniendo como punto de partida que el propósito de este estudio fue de conocer la percepción emocional del estudiante universitario ante el distanciamiento social aplicado debido a la pandemia por COVID19.

De acuerdo a las cifras obtenidas de los estudiantes inscritos en Facpya el campus Cadereyta que cuenta con un total de 332 alumnos los cuales están viviendo la cuarentena en sus hogares, el $47.4 \%$ de ellos en domicilios con entre 4 y 5 personas conviviendo, de los cuales el $46.8 \%$ ha notado cambios en sus estados de humor y lo cual ha originado problemas con las personas con las que convive.

http://transparencia.uanl.mx/

"A pesar de la popularidad, estos datos muestran que, debido al aislamiento, quedarse en casa por varias semanas, meses, ETC, resulta en que los estudiantes vivan con sus familias durante mucho tiempo, por lo que el humor de los estudiantes ha sufrido un cambio negativo". http://transparencia.uanl.mx/

\section{Necesidades sociales de ampliar la oferta educativa}

"Es importante señalar que, si las instituciones de educación superior no comprenden las percepciones y actitudes de los estudiantes hacia este nuevo modelo educativo, si bien este modelo tiene grandes ventajas, también requiere ciertas habilidades y habilidades, si no comprenden la demanda de los estudiantes por la modalidad virtual. Puede encontrar dificultades inesperadas. La participación de los alumnos es más activa.

\section{Investigación sobre la educación virtual.}

La mayoría de estudios han examinado la educación virtual. Exploran la eficacia a través de una serie de criterios, como la satisfacción, retención y el rendimiento.

Un análisis objetivo publicado por la Asociación Americana de Investigación Educativa (¿Cómo se compara la educación a distancia con las clases presenciales en las aulas? Un meta análisis de la Literatura Empírica) examinó la información entre 1985 y 2002.

Los autores analizaron 232 estudios en todos los niveles académicos (K-12 y superiores) que examinan los logros (sobre la base de 57.019 estudiantes), actitud (en base a 35.365 estudiantes) y la retención (basada en 3.744.869 estudiantes) los resultados. Este meta análisis destacó que algunas aplicaciones de la educación en línea son mejores que la enseñanza en clase y algunas eran peores.

Otro memorable análisis a cargo del Departamento de Educación de EE. UU. en 2010. La evaluación de los métodos evidenciados en el aprendizaje en línea: Un meta análisis e inspección de la educación virtual encontraron que, en promedio, los estudiantes de aprendizaje en línea obtuvieron mejores logros, modestamente mejor, a quienes recibieron las clases cara a cara.

"Creo que nos dice que el aprendizaje en línea puede ser eficaz, pero eso por sí solo no es una garantía de que será eficaz. No está 
predeterminado que la educación en línea es mejor (o peor) que un aula tradicional."

\section{Adaptación de la Educación a la Tecnología}

Profesorado y alumnado adoptan nuevas rutinas durante el confinamiento en sus hogares. De repente todas las clases adquieren una dimensión digital y se buscan estrategias para seguir impartiendo la docencia para no perder la programación del curso académico. Es un buen momento para analizar cómo se está trabajando, qué soluciones plantean las empresas tecnológicas y cuáles son los retos de aprendizaje.

La digitalización de la educación no es un tema novedoso. En los últimos años las diferentes etapas del sistema educativo (desde la primaria hasta la universidad) se han ido introduciendo en el universo digital. En la gran mayoría ya se incluyen clases con dispositivos electrónicos (ordenadores y/o tabletas), en algunas se utilizan plataformas tecnológicas de soporte a la docencia y en otras se ofrece la docencia completa.

"Esta nueva situación plantea un reto a todo el sector educativo. Esperemos que los aprendizajes sean mayormente positivos."

\section{Beneficios de la educación en línea}

"Los beneficios de la educación en línea son cada vez más obvios. Por supuesto, todavía existen algunos desafíos, como la calidad de la conexión a Internet, que pueden impedir que maestros bien capacitados y motivados completen los mejores cursos. Otro gran desafío es la aceptación social: muchos padres quieren que sus hijos aprendan exactamente igual que antes. Sin embargo, la cantidad de cursos y programas en línea en México y muchos otros países, así como el crecimiento en la inscripción, están mostrando una aceptación cada vez mayor."

\section{Opinión de Estudiante de FACPyA Campus Cadereyta Lara Torres Brayan Antonio}

En lo personal ha sido algo que de alguna manera me trajo beneficios una de ellas es que reparto mucho mejor mis tiempos ya que anteriormente el hecho de el trayecto que tenia de mi casa a la facultad y viceversa ese tiempo lo aprovecho en otras cosas aparte es mucho más práctico y rápido ya que con el uso de la tecnología es más sencillo hacer llegar la información.

7. Para uno como estudiantes era mucho más óptimo estar en línea puesto que tienes todo al alcance $y$ se facilita todo, pero para ser verdad existen ciertas desventajas puesto que no se logra captar de la misma manera la idea implementada por el maestro

Según Jiménez, “el sistema de aprendizaje es totalmente diferente. En el aula de clase tenemos la ventaja de consultar directamente al docente, pero en las clases virtuales muchas veces esto se complica y quedan dudas sin resolver".

Desde que la humanidad es humanidad, la educación -enseñar y aprender- ha sido presencial. El sabio de la tribu se reunía con los suyos, les daba lecciones de vida e instrucciones para conseguir cómo alimentarse. Todos se reunían para verse, oírse, intercambiar, darse apoyo. Siguieron más tarde los maestros en Oriente. Luego Platón con su academia. Aristóteles con el liceo. 


\section{METODOLOGIA DE LA INVESTIGACION}

La metodología de la investigación que se va a utilizar para comprobar la problemática planteada, se encuentra formada por las siguientes etapas.

- Encuesta aplicada a los docentes

- Encuesta aplicada a los alumnos

\section{1.- DISEÑO DE LA INVESTIGACIÓN}

El estudio de investigación cuenta con un enfoque del tipo cualitativo de exploración, apoyados en datos reales obtenidos directamente de las respuestas de los encuestados.

El estudio cuenta con dos fuentes de información, primaria extraída de la aplicación de las encuestas aplicadas a la muestra obtenida de acuerdo a una prueba de confianza consistente en un total de 332 alumnos.

\section{2.- MUESTRA POBLACIONAL}

La muestra poblacional está compuesto por todos los estudiantes inscritos en FACPyA campus Cadereyta dando un total de 332 estudiantes.

El total de los docentes entrevistados que actualmente imparten clases en la Facultad de Contaduría Pública y Administración Campus Cadereyta son 17.

Los instrumentos de recolección de datos que se utilizarán en dicha investigación consisten en un estudio aplicado a docentes y alumnos los cuales son los que viven la situación actual y así tener información específica de los alumnos de la universidad al respecto de cómo están viviendo en sus hogares la situación de la contingencia por COVID-19.

\section{3.- OBJETIVOS DE LA INVESTIGACIÓN}

Lo que esperamos para esta investigación es saber de qué manera los estudiantes resuelven los problemas a los que se enfrentan durante la pandemia, como manejan a su plataforma de trabajo. 


\section{RESULTADOS}

Resultados de las encuestas aplicadas

Docentes encuestados 16

Equivalente $100 \%$

1. ¿Qué plataforma o recurso utiliza en el proceso de enseñanza aprendizaje durante esta pandemia?

El total de los docentes de FACPYA Cadereyta usa la aplicación de Microsoft Teams sugerida por la UANL.

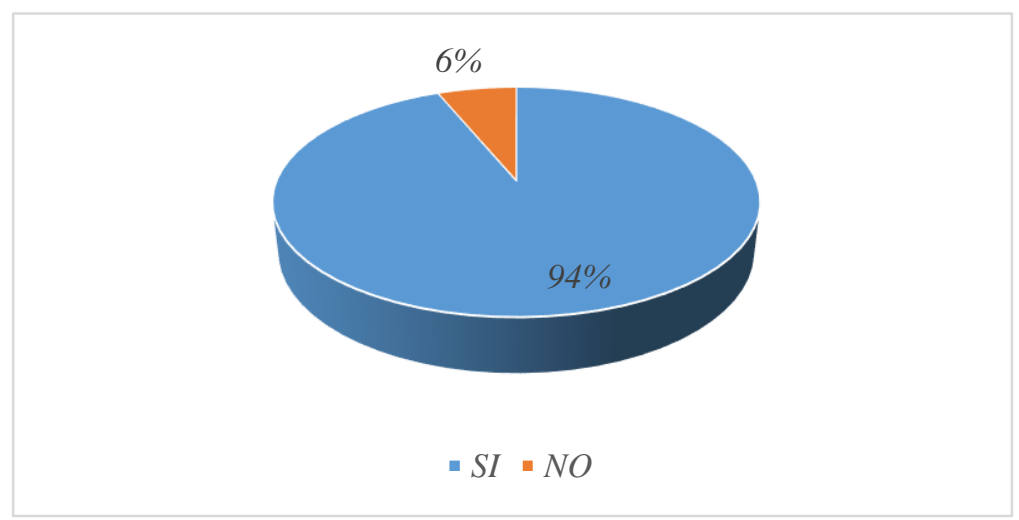

2. ¿Usted considera que los alumnos

se distraen más fácilmente con las clases en línea?

Figura 1. Distracción en los alumnos por clases en línea

El 94\% de los docentes encuestados que equivale a 15/16 consideran y están conscientes de que los alumnos están distraídos por las clases en línea. 
3. ¿Usted considera que los docentes

alumnos que no cuentan con el deben ser más empáticos con los recurso para las clases en línea?

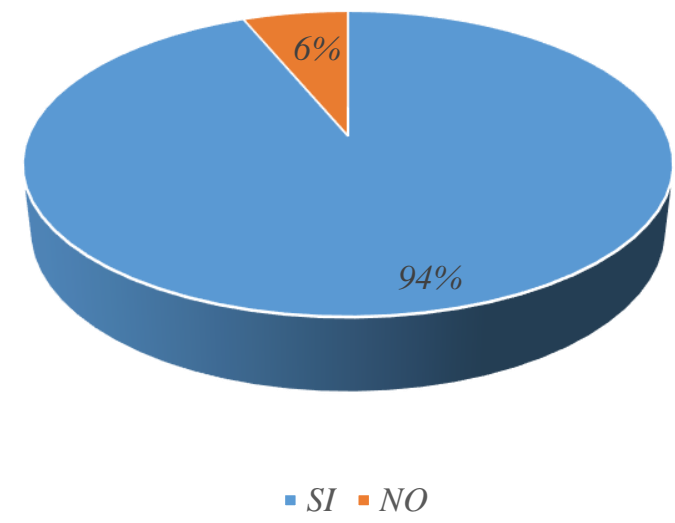

Figura 2. ¿Debería tener empatía del docente hacia el alumnado que no cuenta con recursos para clases en línea?

El $94 \%$ de los docentes en Campus Cadereyta consideran la opción de ser empáticos con sus estudiantes sobre todo con aquellos que no cuentan con el recurso (por el recurso podemos entender que es el dinero para la compra de un dispositivo, el dispositivo como laptop, celular y el dinero para contar con un plan de internet) para llevar a cabo sus clases en línea durante esta pandemia.

4. ¿Es difícil impartir asignaturas prácticas mediante las clases en línea?

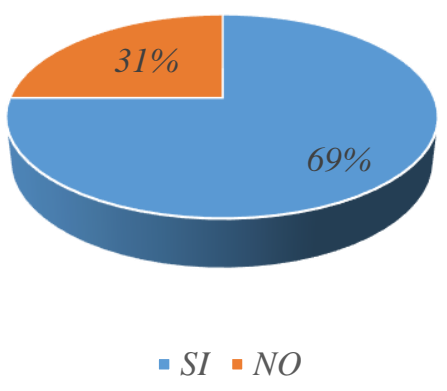

Figura 3. Dificultad en las asignaturas practicas mediante las clases en línea.

El $69 \%$ de los docentes encuestados equivalente a 11 de los 16 están conscientes de lo difícil que es en tiempos de pandemia ver asignaturas prácticas con sus alumnos, algunos de ellos, comentan que tuvieron que modificar la forma de trabajar para que sea más fácil y comprensible para el alumno el aprendizaje de las asignaturas prácticas.

5. ¿Considera usted que el rendimiento de los alumnos ha sido afectado negativamente durante esta pandemia? 


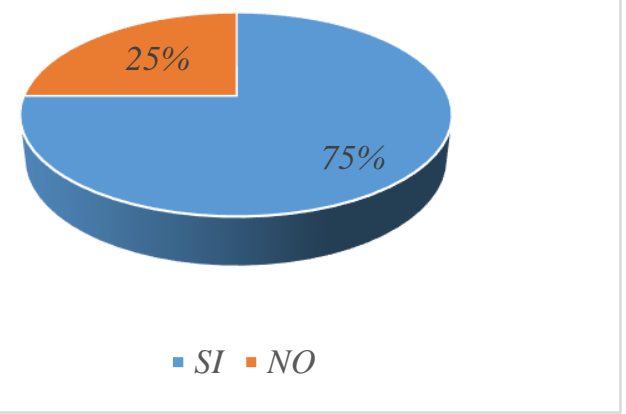

Figura 4. Afectación negativa en el rendimiento de los alumnos durante la pandemia.

La pandemia sin duda ha traído muchos cambios en nuestra sociedad y desde el punto de vista de los docentes del Campus Cadereyta los cuales determinan un $75 \%$ consideran que los alumnos han sufrido cambios negativos en su rendimiento, aprovechamiento y aprendizaje, motivo por el cual como se ve en la pregunta anterior algunos docentes se vieron en la necesidad de modificar su forma de trabajo.

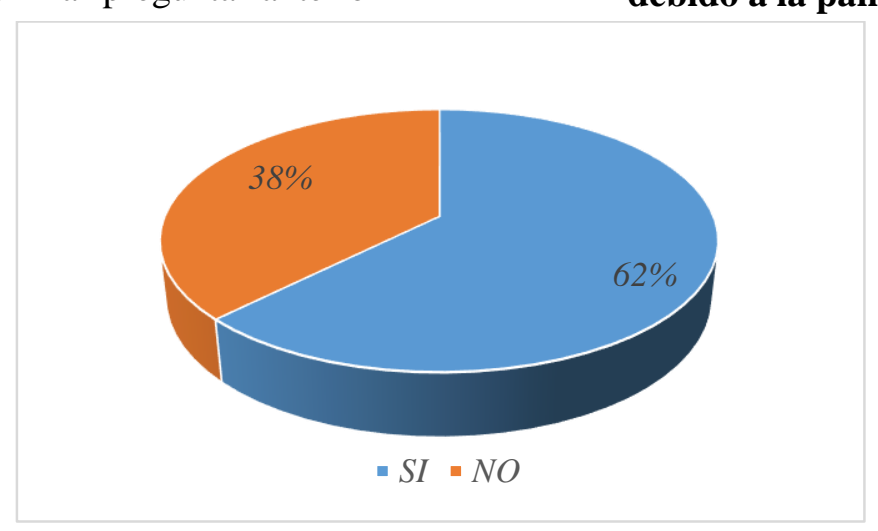

Figura 5. ¿Debería tener empatía del docente hacia el alumnado que no cuenta con recursos para clases en línea?

La respuesta prevaleciente es SI, desde el punto de vista de los docentes del campus Cadereyta los alumnos han sufrido cambios negativos en la conducta como es la apatía ante las clases en línea, la depresión por la situación del confinamiento, la ansiedad de volver a las aulas y el aburrimiento, son los cambios que los docentes consideran que prevalecen en los alumnos durante esta pandemia.

\section{7. ¿Considera usted que ha sufrido cambios emocionales negativos debido a esta pandemia?}




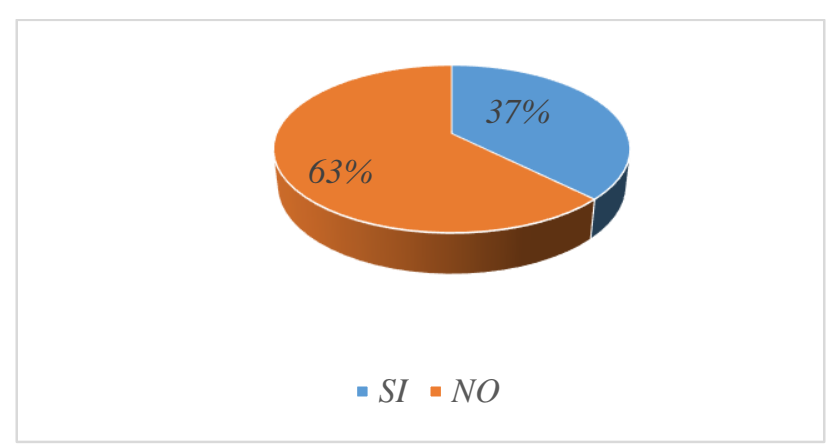

Figura 6. Cambios emocionales negativos a causa de pandemia.

De los 16 docentes encuestados solo 6 de ellos que representan el $37 \%$ de la población del Campus Cadereyta considera que ha sufrido cambios emocionales, y estos cambios son depresión y estrés por el confinamiento y la ansiedad por sentir que la carga de trabajo aumento en estas clases en línea.
8. Si la respuesta anterior fue si, ¿Considera usted que es necesario recibir ayuda para solucionar estos posibles cambios emocionales negativos?

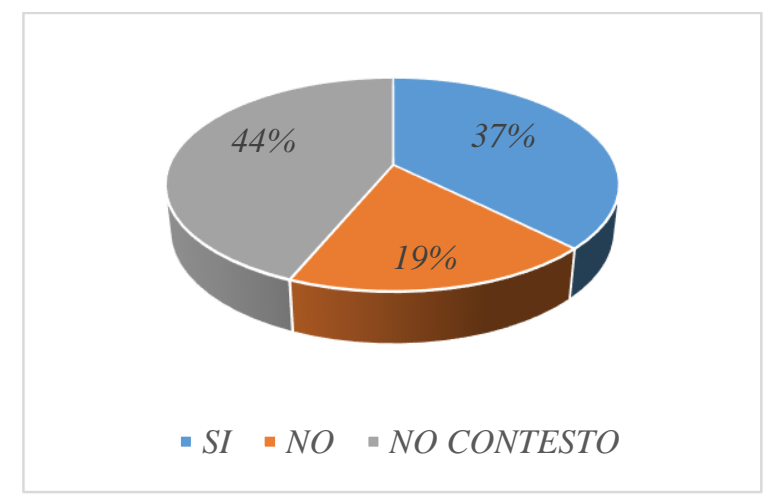

Figura 7. Necesidad de recibir ayuda para solución de cambios emocionales negativos.

De los 6 docentes que contestaron en la pregunta anterior que han sufrido cambios emocionales el total de ellos está dispuesto a recibir alguna ayuda para solucionar sus cambios emocionales, el tipo de ayuda que se puede proporcionar es pláticas con expertos en el tema para ayudar a bajar sus niveles de estrés y depresión ocasionados. 
Encuesta aplicada a los alumnos

Alumnos 332

Alumnos encuestados 303

Equivalente $92 \%$
1. ¿Usted cree que el no contar con los recursos afecta sus clases en línea?

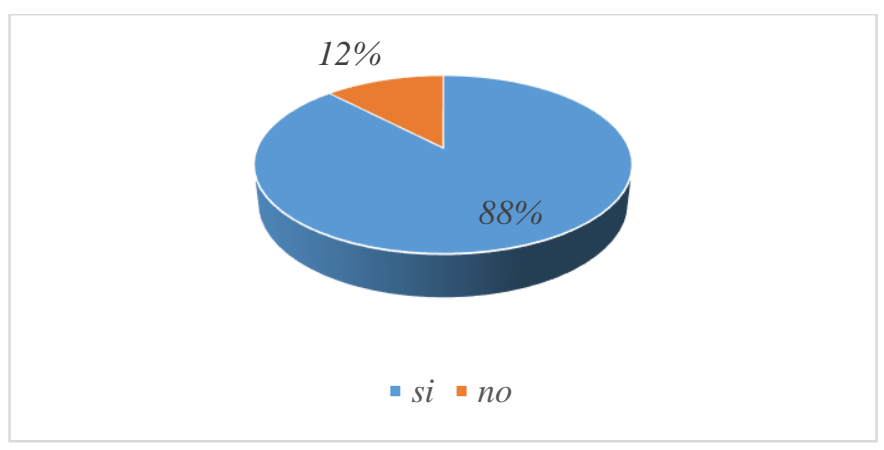

Figura 8. Afectación por no contar con los recursos para clases en línea.

Indiscutiblemente el $88 \%$ de los alumnos consideran que el no contar con los recursos para tomar las clases en línea es un problema, y definitivamente lo es debido a que para tener el adecuado aprendizaje es necesario por lo menos en tiempos de pandemia la conexión a internet para estar en las clases y por lo menos ver las grabaciones de las clases.

\section{2. ¿Cuál es su experiencia de trabajar en la plataforma MS Teams?}

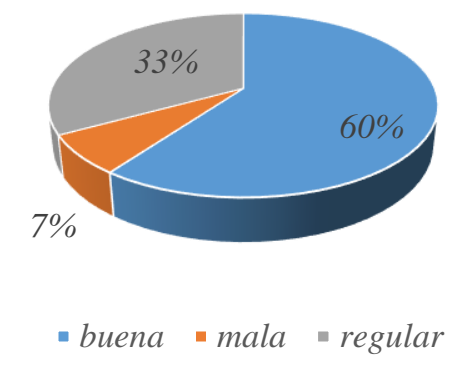

Figura 9. Experiencia de trabajar en la plataforma MS Teams

En la UANL la plataforma Microsoft es regular y solo un $7 \%$ de los alumnos consideran que la plataforma es muy mala.

Teams es la que actualmente en tiempos de pandemia está siendo usada en la cual el $60 \%$ de los alumnos consideran que su experiencia es buena, el 33\% considera que la experiencia

3. ¿Usted cree que afecto su rendimiento escolar el tomar actualmente clases en línea? 


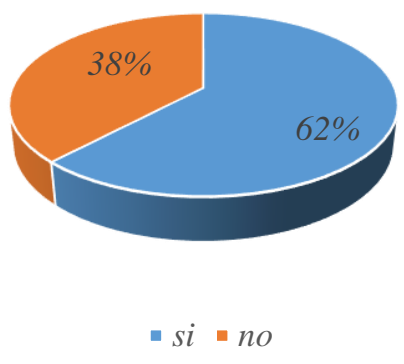

Figura 10. Afectación en el rendimiento escolar al tomar clases en línea

El $62 \%$ de los alumnos encuestados consideran que su rendimiento se ha visto afectado negativamente en tiempos de pandemia, lo cual es comparable con la percepción de los docentes los cuales también opinan que se ha visto afectado su rendimiento.

\section{4. ¿Cuál es la percepción que tiene de las asignaturas prácticas en línea actualmente?}

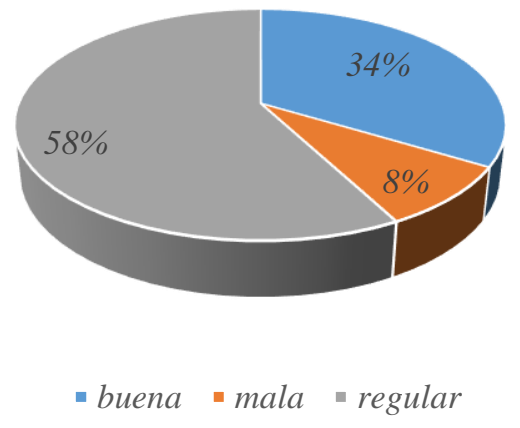

Figura 11. Percepción hacia las asignaturas prácticas en línea actualmente.

Gracias a que los docentes de las asignaturas prácticas del Campus Cadereyta modificaron su forma de trabajo la percepción de los alumnos fue buena respecto a esta situación exactamente un $58 \%$ de los encuestados, por lo tanto, solo el $8 \%$ tiene una mala percepción de las asignaturas practicas durante estas clases en línea. 


\section{5. ¿Qué es lo que más extraña de las clases presenciales?}

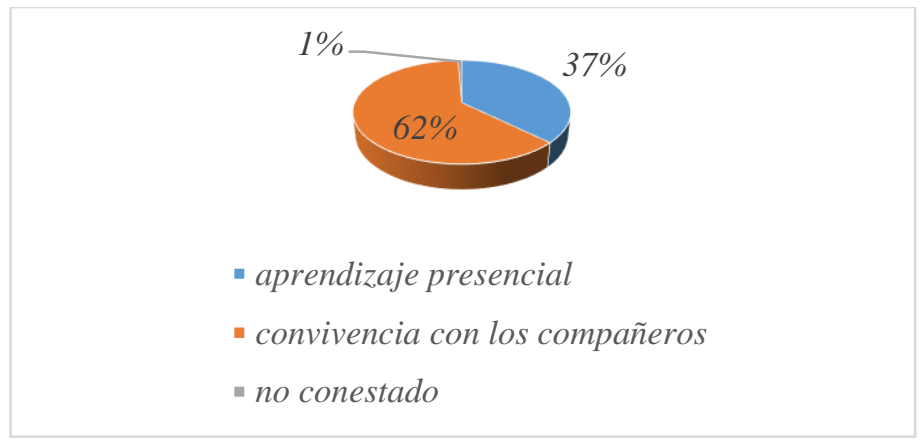

Figura 12. Razón por la cual extrañan de clases presenciales

Sin duda la mayoría de las personas extrañan la vida antes de la pandemia, los alumnos encuestados del Campus Cadereyta extrañan el $62 \%$ la convivencia con sus compañeros en las aulas, el $37 \%$ el

\section{6. ¿Usted considera que los docentes están preparados para impartir clases en línea?}

aprendizaje obtenido en las aulas.

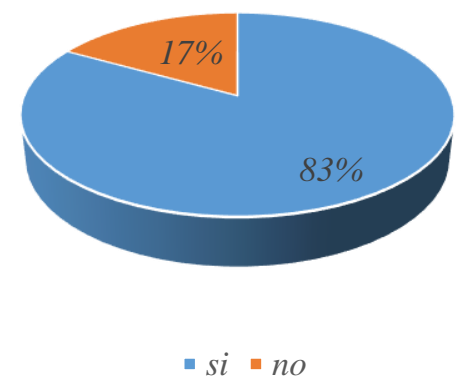

Figura 13. Consideración de la preparación de docentes en impartir clases en línea.

El $100 \%$ de los docentes del Campus Cadereyta están preparados para impartir clases en línea y de hecho están actualizados en el uso de las plataformas sobre todo la plataforma usada por la UANL, sin embargo, la percepción de los alumnos encuestados es apenas del $83 \%$. 


\section{7. ¿Usted prefiere?}

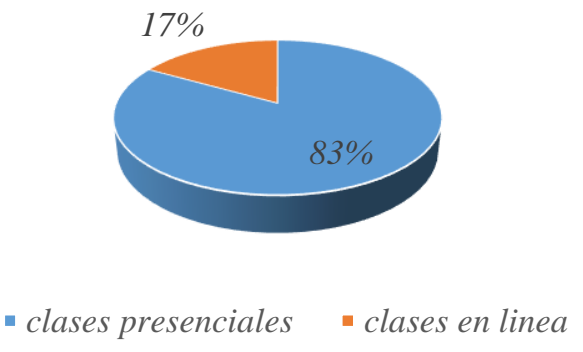

Figura 14. Preferencia de clases presenciales o clases en línea.

El tener clases en línea tiene ventajas encuestados prefieren estar en clases como todos sabemos, las cuales son el evitar presenciales.

el traslado de la casa del alumno a la escuela y el gasto mismo que se incurre, sin embargo, no es suficiente por lo que los alumnos del Campus Cadereyta consideran que ya es tiempo de regresar y el $83 \%$ de los

\section{8. ¿Usted considera que ha tenido cambios emocionales y de conducta durante esta pandemia?}

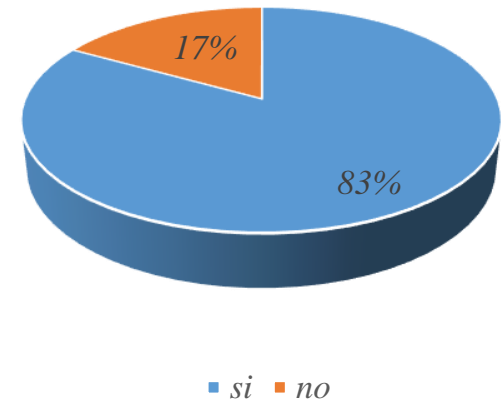

Figura 15. Consideración de cambios emocionales y de conducta durante la pandemia.

La situación del confinamiento ha generado diversos cambios emocionales en la mayoría de las personas, en el Campus Cadereyta el $83 \%$ de los alumnos encuestados consideran que han sufrido cambios como depresión, ansiedad y estrés. 


\section{CONCLUSIONES}

Dentro de este trabajo de investigación se dio a conocer el concepto de gamificación, en cual se presenta una explicación abundante del tema además de agregar contenido de grandes autores.

Como puede observarse en base a los resultados de las encuestas aplicadas a docentes y a estudiantes se llega a la conclusión única que a pesar de no estar relacionados al $100 \%$ con las clases en línea los alumnos estarían dispuestos a dar lo mejor de ellos para obtener buenos resultados .

La mayor parte de los docentes están dispuestos a implementar técnicas nuevas en sus unidades de aprendizaje, e incluso están dispuestos a capacitarse en estos nuevos temas que se desea implementar en las aulas virtuales del Campus Cadereyta.

De acuerdo con la investigación realizada sobre la situación sanitaria actual referente al COVID-19 el incremento del estrés en los alumnos fue muy significativo, además de ocasionar frustración y ansiedad, esto no solo es un evento que solo aplique para alumnos, sino también para docentes y padres de familia, hablando ya de la educación básica, media superior y superior.
Evaluando la situación de crisis del cual se avanzó poco a poco, todo entorna a un mismo sitio, uno de los panoramas el cual nos ayudara a llevar a cabo todos los propósitos establecidos con el fin de poder encontrar la forma más "fácil", dinámica, de enseñanza y aprendizaje, además de buena comunicación del docente con el alumno e inversa.

Por último se concluyó que aunque al principio de la pandemia y después el regreso oficial a clases en línea, muchos docentes no se adaptaban a la plataforma por lo cual estarían impartiendo sus clases, ha esto sumándole la falla de conexión a internet, no tener un equipo de cómputo propio, también la falta comunicación y el estrés de no poder llevar el control de las situaciones por fallas ajenas incremento el estrés y esto ocasiono el bajo rendimiento de alumnos y docentes, el cual se detectó y se encontró la estrategia para poder llevar a cabo de una manera más dinámica, cumpliendo con las competencias establecidas en cada docente.

De igual forma, es el caso considerar que, nadie estábamos preparados para una pandemia, sin embargo, en base a las estrategias y análisis se ha podido cumplir con el objetivo, de poder retomar las clases y poder cumplir con las obligaciones tanto docentes y alumnos, con el resguardo de su salud. 


\section{1.- RECOMENDACIONES}

Las recomendaciones que se pueden agregar en este trabajo de investigación son sencillas y fáciles de implementar las cuales consisten en:

- Capacitar a la totalidad de los docentes en el tema de llevar de manera adecuada las en línea:

Para llevar a cabo esta recomendación será necesario impartir un curso donde la idea es dar a conocer el tema de la plataforma que la institución utilice y diseñar un proceso de implementación de tal concepto.

- Adecuar los programas de cada unidad de aprendizaje para implementar actividades con el proceso clases en línea:

Para esto es necesario reunirse con los encargados de la elaboración de los programas de cada unidad de aprendizaje y comentarles del nuevo proceso para llevar a cabo los cambios necesarios en los mismos.

- Dar a conocer a los alumnos de la nueva implementación del proceso de enseñanza:

Esta recomendación es sencilla y fácil de aplicar debido a que los propios alumnos están dispuestos a recibir un nuevo proceso de enseñanza -aprendizaje en línea debido a los cambios tecnológicos que se está enfrentando la sociedad.

- Motivar a los alumnos a participar en el proceso de utilizar la tecnología:

- Al igual que la recomendación anterior para llevar a cabo esto solo basta con dar a conocer los cambios. Motivar a los alumnos a través de clases dinámicas: para llevar a cabo esto solo es necesario impartir clases dinámicas, interactivas para los alumnos, donde se involucren, tanto en participación como en las actividades aplicadas, ya que no solo es un, es de manera plural por los que estamos pasando por esta situación.

- Comprender las situaciones de cada estudiante, ser empáticos

- Para poder llevar a cabo esta recomendación, solo se necesita ser empático con la gente, ya que todos estamos pasando por una pandemia y no todos responden de la misma manera que otros.

- Contar con pláticas con expertos en el tema para que les ayuden en cuanto a sus cambios emocionales 


\section{REFERENCIAS}

Andrea, O. (11 de Abril de 2020). ¿Qué opinan los estudiantes caleños de las clases virtuales? El país.

Ariadna, O. (2020). Educación en tiempos de covid-19. Mexico: Expansión politica.

Beryona, J. (2020). Clases virtuales. El heraldo, Pagina inicial.

Borrero, J. (2020). Es la educación virtual buena o mala? Educación Virtual Inicial.

Carmina, C. (04 de Abril de 2020). Todas las clases 'on line': ¿cómo nos estamos adaptando? EL periódico

Cervantes, F. (2017). Y tú... ¿qué piensas del aprendizaje en línea? Mexico: Vol. 18.

Erika, G. (08 de Agosto de 2020). El reto de la educación a distancia. El sol de México , pág. pagina inicial.

Mejía Rueda, D. (2020). Las clases en linea. El Universo.

Mundo, B. (2015). Por qué las escuelas por internet son "peores que las tradicionales". BBC, 18-27.

Olcese, D. (2021). El panorama del aprendizaje en línea en México. Mexico: Forbes.

Paz, A. (2020). percepción emocional. Revista médica, pagina inicial.

Sanabria Mesa, A. L. (2013). Revista Fuentes, 117-138.

Sandoval, J. (2020). Opinión de estudiantes sobre el uso de apoyos didácticos en línea en un curso universitario. Scielo, 165-175.

School, I. (2020). Estudiar a distancia, ventajas y opiniones. IMF.

Vanguardia, L. (2020). Rendimiento academico tiempos de covid. 6154585.

Velazquez, G. (2020). Estres academico en estudiantes universitario asociados a la pandemia por COVID-19. Innovación mas desarrollo, 9-25.

Villafuerte. (2020). Educación en tiempos de pandemia. Tecnologico de Monterrey: Observatorio de innovavión educaativa. 